\section{Hippocrates, Still, and Osler: A shared philosophy}

Hippocratic philosophy and principles underlie basic osteopathic medical philosophy. George W. Northup, DO, wrote in Osteopathic medicine: An American reformation, "While the Hippocratic school emphasized the study of the health of man as an individual, integrated unit, the rival school at Cnidus, a nearby peninsula, steered its attention away from man toward disease, the afflictor of man.... Basically, osteopathic medicine and its physiological principles are supporters of the Hippocratic approach. Although it borrows from both Hippocratic and the Cnidian, the emphasis is clearly on Man rather than the individual diseases that afflict man."1

See related article on page 1243 .

Like Thomas Sydenham and Hermann Boerhaave before him and Sir William Osler after him, Andrew Taylor Still incorporated the Hippocratic thought and philosophy into his own. While there are remarkable similarities between Still and Hippocrates' philosophy, Still apparently did not specifically acknowledge the latter's influence in any of his writings.

In "The rebirth of Hippocratic thought," beginning on page 1243 in this issue, Robert E. Suter, examines the similarities between Still and Hippocrates. He argues that Still was influenced more directly by Hippocrates' teaching than previously recognized. With that in mind, the reference to Hippocrates' special attention to the musculoskeletal system and his description of manipulative approaches to musculoskeletal disorders proves particularly interesting.
Suter draws striking parallels between Still and Osler. These contemporary giants, who apparently never met, developed philosophies similarly influenced by Hippocratic principles. Concludes Suter, "In that teachings of both William Osler and Andrew Taylor Still are to a large extent consistent with those of Hippocrates, the view that American medicine today reflects the influence of these two men can also been seen as a belated return to and rebirth of Hippocratic thought and medicine." THOMAS WESLEY ALLEN, DO, FACOI

1. Northup, GW: Osteopathic medicine: An American reformation. Ed 2, p 4. American Osteopathic Association, Chicago, 1979. 


\section{Home IV Antibiotic Therapy: An Introduction}

Lowering health care costs while maintaining high quality care is a goal that all physicians strive for. An important step toward this goal is the development of home health care that is both therapeutically and economically effective. One particular aspect of home health care that is gaining acceptance is home intravenous antibiotic therapy.

\section{Evolution of an Industry}

Experts anticipate that over the next several years home IV antibiotic therapy will be one of the fastest growing segments of the home health care market. Today, physicians have access to an industry that provides necessary technology, personal services and pharmaceutical supplies - an industry devoted to home health care. With the growth of this industry, most physicians will be able to successfully manage many patients with a minimum of acute hospital care.

\section{The Home IV Therapy Decision}

Almost any patient who requires IV antibiotic therapy, and is hospitalized to receive that therapy, could be considered for treatment at home. However, four criteria are often applied before implementing home IV therapy: 1

1. No suitable oral therapy is available.

2. The patient's condition is stable enough for discharge, and the patient can monitor his/her own therapy.

3. The patient agrees to outpatient therapy after a full disclosure of responsibilities and potential problems.

4. The patient has a suitable home environment, including a phone for emergency communications and a refrigerator for antibiotic storage.

Any infection that responds to IV therapy can potentially be treated at home, including osteomyelitis, endocarditis, wound infections, urinary tract infections, septic arthritis and others. ${ }^{2}$

Many classes of antibiotics appear suitable for home IV infusion; however, antibiotics used in outpatient care should have low toxicity, a broad spectrum of activity and a long half-life, allowing for less frequent dosing. Antibiotics with long half-lives enhance the convenience and cost effectiveness of home therapy and facilitate patient compliance.

\section{Advantages}

Home IV antibiotic therapy removes the risk of nosocomial infection. Receiving therapy at home in warm and familiar surroundings also provides psychological benefits. Another benefit is that patients in the home care environment become more active in their recovery. These intangibles appear to contribute to more rapid and predictable recovery.

There are also considerable financial advantages to home IV antibiotic therapy. For those patients who are able to work while receiving therapy, the benefits are obvious. Of course, savings are also accrued because patients are not hospitalized for long periods of time. In one study, the calculated savings ranged from $\$ 2,791$ to $\$ 4,651$ per patient. $^{3}$

In addition, reimbursement for home services has greatly improved during the past five years. Many insurers now pay for outpatient therapy that can replace treatment given in the hospital. Congress has also provided for such reimbursement in the Medicare population under the catastrophic health insurance law that was recently enacted.

The success of home IV antibiotic therapy depends on a team effort employing sound case management by the physician and quality service from the home health care industry. Teamwork by these two groups will help ease the medical profession into a new era of health care.

\section{References}

1. Larson SE: Home IV antibiotic therapy: The primary-care physician's role. Drug Therapy, Nov 1987:67-74.

2. Rising JB: Home IV antibiotic therapy: Roles you can play. U.S. Pharmacist, Oct 1987:H9-H14.

3. Graves G, Jackson JP, Maxwell A, Woods T: Home intravenous antibiotic therapy in Arkansas. J Arkansas Med Soc 1987;84:55-57. 


\section{In every small town, you'll find a church, an American flag, and someone who'll sell you cocaine.}

It's a sad fact, but a fact nonetheless. Substance abuse is so widespread these days that buying cocaine is almost as easy as buying a hot dog or a new pair of shoes.

As a physician, you're in the unique position to detect drug abuse in your patients, using your training, thorough work-ups, and advanced diagnostic techniques.

Through increased detection, you can help curb this growing problem, and help make our towns (both small and large) a home for people, not pushers.

Looking for drug abuse-make it a habit. Partnership for a Drug-Free America

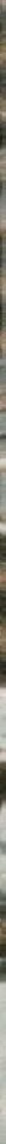




\section{Who's taking MEVACOR'?}

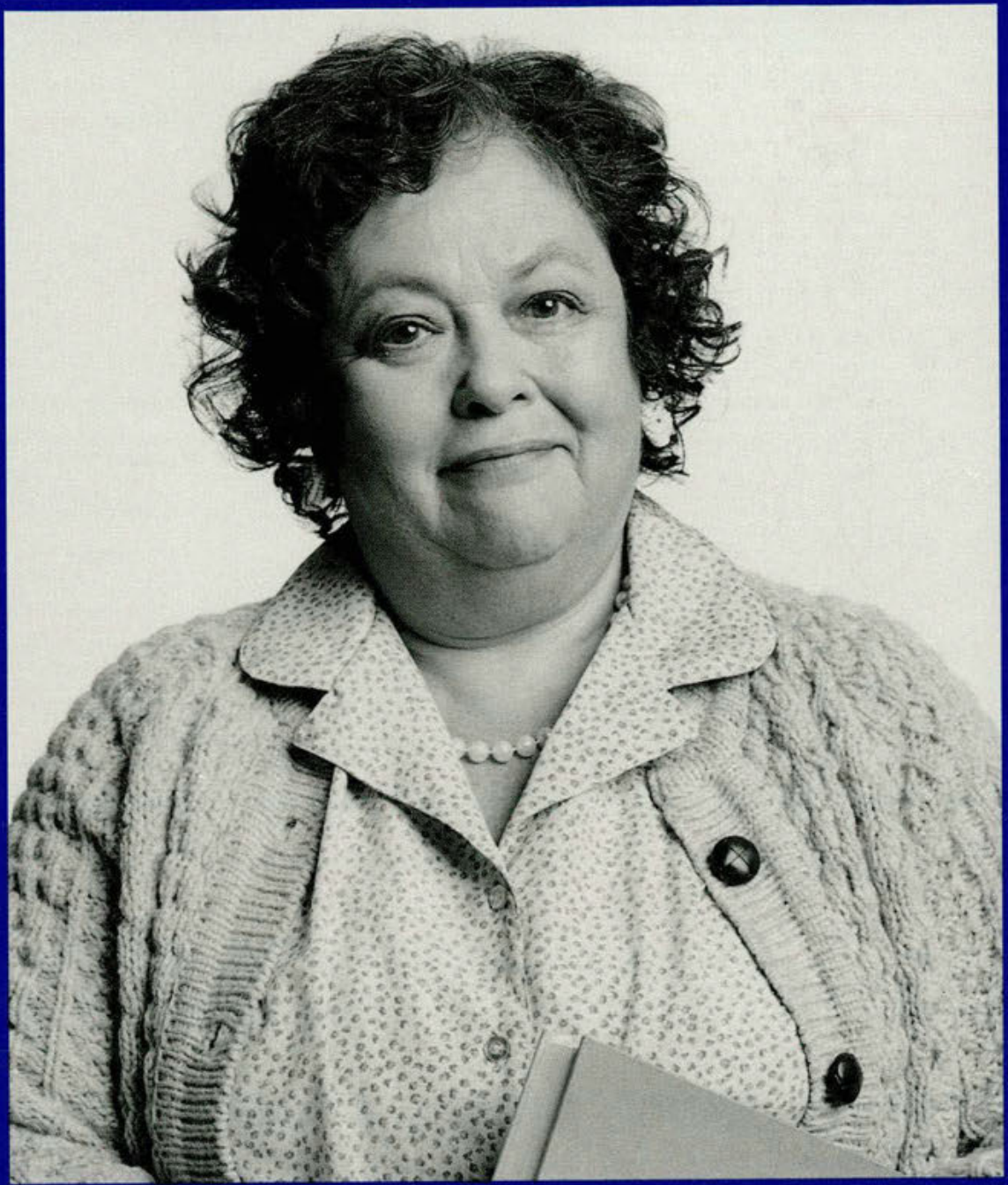

MEVACOR is indicated as an adjunct to diet for the reduction of elevated total and LDL cholesterol levels in patients with primary hypercholesterolemia (Types $\mathrm{Ila}$ and $\mathrm{IIb}$ ) when response to nonpharmacologic measures has been inadequate

MEVACOR is contraindicated in patients who are hypersensitive to any component of the medication. in patients with active liver disease or unexplained persistent transaminase elevations; in pregnant or lactating patients: and in women of childbearing age, except when such patients are highly unlikely to conceive.

It is recommended that liver function tests be performed before treatment begins, every 4 to 6 weeks during the first 15 months of therapy, and periodically thereafter in all patients

The effect of lovastatin-induced changes in serum lipoprotein levels, including reduction of serum cholesterol. on cardiovascular morbidity or mortality has not been established

For more details on warnings, precautions, and adverse reactions, including cautionary information regarding liver dysfunction, myopathy, and slit-lamp monitoring. please see Prescribing Information

For a Brief Summary of Prescribing Information, please see the following page.

\section{Patient L.J.K.}

-Female, age 56

-Elevated total cholesterol of $268 \mathrm{mg} / \mathrm{dL}$ after adequate trial of a weight-loss and cholesterol-lowering diet

-Obese-more than 30\% above ideal weight

- Has cut down to one pack of cigarettes a day

-After first 6 weeks on MEVACOR, total cholesterol is down to $220 \mathrm{mg} / \mathrm{dL}$ (dosage: $20 \mathrm{mg}$ once daily with the evening meal)

-Dosage increased to 20 mg b.i.d.; after 6 additional weeks, total cholesterol is down to $196 \mathrm{mg} / \mathrm{dL}$

\section{Results with MEVACOR}

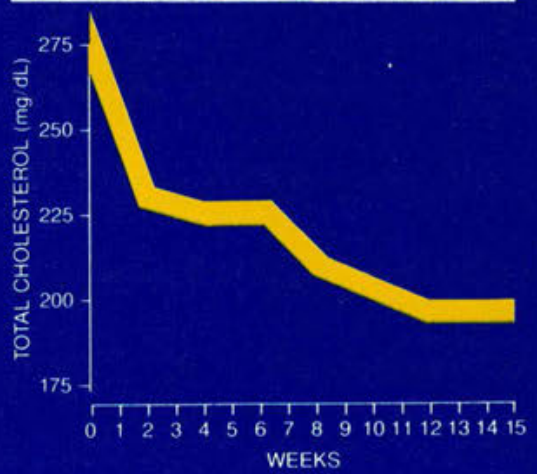

Not everyone will respond as did the patient in this hypothetical case history: however, the mean reduction in total cholesterol in clinical trials was $17 \%$ at $20 \mathrm{mg}$ once a day and $27 \%$ at $20 \mathrm{mg}$ b.i.d 


\section{MEVACOR}

CONTRAINDICATIONS

Hypersensitivity to any component of this medication.

Active liver disease or unexplained persistent elevations of serum ransaminases.

Pregnancy and lactation.

Atherosclerosis is a chronic process and the discontinuation of lipidlowering drugs during pregnancy should have little impact on the outcome terol and other products of the cholesterol biosynthesis pathway are essential components for fetal development, including synthesis of steroids and cell membranes. Because of the ability of inhibitors of HMG COA reductase such as MEVACOR* (Lovastatin. MSD) to decrease the synthesis of cholesterol and possibly other products of the cholesterol synthesis of cholesterol and possibly other products of the cholestero
biosynthesis pathway. MEVACOR may cause fetal harm when administered to a pregnant woman. Therefore. lovastatin is contraindicated during pregnancy. Lovastatin should be administered to women of childbearing age only when such patients are highly unlikely to conceive. If the discontinued and the patient should be apprised of the potential hazard to

\section{WARNINGS}

Marked persistent increases (to more than 3 times the upper limit of normal) in serum transaminases occurred in $1.9 \%$ of adult patients who received lovastatin for at least one year (see ADVERSE REACTIONS) When the drug was interrupted or discontinued in these patients, the transaminase levels usually fell slowly to pretreatment levels. The increases usually appeared 3 to 12 months after the start of therapy with ovastatin and were not associated with jaundice or other clinical signs or symptoms. There was no evidence of hypersensitivity. A liver biopsy was done in one of these patients and showed areas of focal hepatitis. In this patient, transaminase levels returned to normal following discontinuation of therapy. Some of these patients had abnormal liver function tests prior to lovastatin therapy and/or consumed substantial quantities of alcohol It is recommended that liver function tests be performed before treatment begins, every 4 to 6 weeks during the first 15 months of therapy with lovastatin, and periodically thereafter in all patients. Special attention should be paid to patients who develop elevated serum transaminase levels, and in these patients, measurements should be repeated promptly and then performed more frequently. If the transaminase levels show evidence of progression, particularly if they rise to 3
times the upper limit of normal and are persistent, the drug should be times the upper limit of normal and are persistent, the drug should be
discontinued. Liver biopsy should be considered if elevations are persistent beyond the discontinuation of the dru

The drug should be used with caution in patients who consume substantial quantities of alcohol and/or have a past history of liver disease.
Active liver disease or unexplained transaminase elevations are contraindications to the use of lovastatin.

As with other lipid-lowering agents, moderate (less than 3 times the upper limit of normal) elevations of serum transaminases have been reported following therapy with MEVACOR (see ADVERSE REACTIONS). These changes appeared soon after initiation of therapy with MEVACOR, were often transient, were not accompanied
interruption of treatment was not required.

\section{Skeletal Muscle}

Myalgia has been associated with lovastatin therapy. Transient, mildly elevated creatine phosphokinase levels are commonly seen in lovastatintreated patients. However, in clinical trials, approximately $0.5 \%$ of patients developed a myopathy, i.e., myalgia or muscle weakness associated with markedly elevated CPK levels. In clinical trials and since the drug was marketed, there have been rare reports of severe rhabdomyolysis that precipitated acute renal failure, especially in cardiac transplant patients on immunosuppressive therapy including cyclosporine. Myopathy should be considered in any patient with diffuse myalgias, muscle tenderness or weakness, and/or marked elevation of CPK. Patients should be advised to report promptly unexplained muscle pain, tenderness, or weakness. be discontinued if markedly elevated CPK levels occur or myopathy is diagnosed.

Most of the patients who have developed myopathy while taking lovastatin were receiving concomitant therapy with immunosuppressive drugs, gemfibrozil, or lipid-lowering doses of niacin (nicotinic acid). In clinical trials, about $30 \%$ of patients on concomitant immunosuppressive
therapy including cyclosporine developed myopathy; the corresponding therapy including cyclosporine developed myopathy; the corresponding
percentages for gemfibrozil and niacin were approximately $5 \%$ and $2 \%$, percentages for gemfibrozil and niacin were approximately $5 \%$ and $2 \%$,
respectively. It is not known whether the same phenomenon occurs with fibrates other than gemfibrozil. Therefore, the benefits and risks of using lovastatin concomitantly with immunosuppressive drugs, fibrates, of lipid-lowering doses of niacin (nicotinic acid) should be carefully

In 6 patients with cardiac transplants taking immunosuppressive therapy including cyclosporine concomitantly with lovastatin $20 \mathrm{mg} /$ day, the average plasma level of active metabolites derived from lovastatin was elevated to approxip 4 ines the expected levels. Because of an lites derived from lovastatin and myopathy, the daily dosage in patients taking immunosuppressants should not exceed 20 mg/day in patients AGE AND ADMINISTRATION) Even at this dosage, the benefits and risks of using lovastatin in patients taking immunosuppressants should be

Consideration should be given to temporarily withholding or discontinuing drug therapy in any patient with a risk factor predisposing to the development of renal failure secondary to rhabdomyolysis, including: severe acute infection, hypotension, major surgery, trauma, severe met-
abolic, endocrine, or electrolyte disorders, and uncontrolled seizures.

\section{PRECAUTIONS}

Before instituting therapy with MEVACOR, an attempt should be made to control hypercholesterolemia with appropriate diet, exercise, weight reduction in obese patients, and to treat other underlying medical problems (see INDICATIONS AND USAGE).

Eye

There was a high prevalence of baseline lenticular opacities in the patient population included in the clinical trials with lovastatin. During relationship of lovastatin to these findings has not been established. Of 431 patients examined with slit lamp at baseline and during therapy with lovastatin, 34 had opacities reported at the final examination ( 5 to 15 months after starting lovastatin) that were not noted at baseline. On the
other hand, in 45 patients, opacities observed at baseline were not noted at the final examination, so that the prevalence did not increase. There was no clinically significant change in visual acuity in the patients who had new opacities reported, nor was any patient, including those with opacities noted at baseline, discontinued from therapy because of a decrease in mended that patients placed on lovastatin therapy be examined with a slit mended that patients placed on lovastatin therapy be examined with a slit
lamp before or shortly after initiation of treatment and annually thereafter. Homozygous familial Hypercholesterolemia

MEVACOR* (Lovastatin, MSO) is less effective in patients with the rare homozygous familial hypercholesterolemia, possibly because these patients have no functional LDL receptors. MEVACOR appears to be more homozygous patients.

Drug Interactions

Immunosuppressive Drugs, Gemfibrozil, Niacin (Nicotinic Acid): See WARNINGS, Skeletal Muscle

Coumarin Anticoagulants: In a clinical trial in warlarin-treated patients designed specifically to observe a potential effect of lovastatin on the pro. thrombin time, lovastatin in dosages up to $40 \mathrm{mg}$ b. .i.d. did not produce any the drug was marketed, clinically evident bleeding and or increased prothrombin time have been reported in a few patients taking coumarin anticoagulants concomitantly with lovastatin. The causal relationship to lovastatin is unclear Nevertheless, it is recommended that in patients taking anticoagulants, prothrombin time be determined before starting lovastatin and frequently enough during early therapy to insure that no significant alteration of prothrombin time occurs. Once a stable prothrom significant alteration of prothrombin time occurs. Once a stable prothromintervals usually recommended for patients on coumarin anticoagulants. II intervals usually recommended for patients on coumarin anticoagulants. If Lovastatin therapyhas notbeen associated with bleeding or with changes in prothrombin time in patients not taking anticoagulants

Antipyrine: Antipyrine is a model for drugs metabolized by the mic: somal hepatic enzyme system (cytochrome P450 system). Becaus lovastatin had no effect on the pharmacokinetics of antipyrine, interacPropranolol: In normal volunteers, there was no clinically significant Propranolol: In normal volunteers, there was no clinically significant
pharmacokinetic or pharmacodynamic interaction with concomitant adpharmacokinetic or pharmacodynamic interaction with co

ministration of single doses of lovastatin and propranolol.
Digoxin: In patients with hypercholesterolemia, concomitant admin. istration of lovastatin and digoxin resulted in no effect on digoxin plasma concentrations.

Other Concomitant Therapy: Although specific interaction studies were not performed, in clinical studies, lovastatin was used concomitantly with beta blockers, calcium channel blockers, diuretics, and nonsteroidal antiinflammatory drugs (NSAIDs) without evidence of clinically significant

Drug/laboratory Test Interaction

Lovastatin may elevate creatine phosphokinase and transaminase levential diagnosis of Carcinogenesis, Mutagenesis, Impairment of Fertility

In a 21-month carcinogenic study in mice, a statistically significan $(p<0.05)$ increase in the incidence of hepatocellular carcinomas and adenomas was observed at doses of $500 \mathrm{mg} / \mathrm{kg} /$ day ( 312 times the not seen in mice given doses of 20 and $100 \mathrm{mg} / \mathrm{kg} / \mathrm{day}(12.5$ and 62.5 mes the maximum recommended human dose).

A statistically significant increase $(p<0.05)$ in the incidence of pulmonary adenomas was seen in fermale mice receiving $500 \mathrm{mg} / \mathrm{kg} / \mathrm{day}$ (312 times the maximum recommended human dose); no similar changes
were seen in males at any dose or in females receiving 20 or 100 were seen in males at any dose or in fernales receiving 20 or 100
$\mathrm{mg} / \mathrm{kg} / \mathrm{day}$ (12.5 or 62.5 times the maximum recommended human $\mathrm{mg} / \mathrm{kg} /$ day $(12.5$ or 62.5 times the maximum recommended human of untreated animals in studies of similar duration, the relationship of this of untreated animals in studies of similar

In addition, an increase in the incidence of papilloma in the nonglandular mucosa of the stomach was observed in mice receiving 100 and $500 \mathrm{mg} / \mathrm{kg} /$ day (62.5 and 312 times the maximum recommended human cose); no increase was seen at a dosage of $20 \mathrm{mg} / \mathrm{kg} / \mathrm{day}$ ( 12.5 times the maximum recommended human dose). The glandular mucosa was not tantly, there is a strong association between this change and hyperplasia tantly, there is a strong association between this change and hyperplasia of the squamous epithelium (acanthosis) in this region; acanthosis is a treated with HMG-COA reductase inhibitors and is most probably a result treated with HMG-COA reductase inhibitors

Similar squamous epithelium is found in the esophagus and anorectal
Silion the reductase in this tissue. unction of the mouse and rat; however, no evidence of a similar drugnduced hyperplastic response was observed in these tissues in studies of up to 21 months in the mouse given up to $500 \mathrm{mg} / \mathrm{kg} / \mathrm{day}$ ( 312 times the maximum recommended human dose), or in a study of 24 months in
the rat given $180 \mathrm{mg} / \mathrm{kg} /$ day (112 times the maximum recommended
human dose)

In a 24-month carcinogenicity study in rats, there was a positive dose esponse relationship for hepatocellular carcinogenicity in males (unadusted $p=0.025$ ). However, because the incidence of hepatocellular carcinogenicity observed in male rats in this study is similar to that observed
spontaneously in this strain of rat. the implications of this finding are spontan
unclear.

No evidence of mutagenicity was observed in a microbial mutagen test using mutant strains of Salmonella typhimurium with or without rat or mouse liver metabolic activation. In addition, no evidence of damage to genetic material was noted in an in vitro alkaline elution assay using rat or mouse hepatocytes, a V-79 mammalian cell forward mutation study. an in
vitro chromosome aberration study in CHO cells, or an in vivo chrovitro chromosome aberration study in $\mathrm{CHO}$ cells.

No drug-related effects on fertility were found in studies with rats.

Pregnanc

Pregnancy Category $X$

SEe CONTRAINDICATIONS

Lovastatin has been shown to produce skeletal malformations in the rat fetus at doses of $800 \mathrm{mg} / \mathrm{kg} /$ day ( 500 times the maximum recommended human dose). At similar doses in mice, an increase in skeletal malformations was observed. These individual changes are within the range of those observed spontaneously in this strain of mouse. No drug-induced changes were seen in either species at doses of up to $80 \mathrm{mg} / \mathrm{kg} / \mathrm{day}(50$
times the maximum recommended human dose) No evidence of malfortimes the maximum recommended human dose). No evidence of malformations was noted in rabbits at up to $15 \mathrm{mg} / \mathrm{kg} / \mathrm{day}$ (highest tolerated no data in pregnant women.

Nursing Mothers

Studies in rats have shown that lovastatin is excreted in the milk. It is not known whether this drug is excreted in human milk. Because many drugs are excreted in human milk and because of the potential for serious adverse reactions in nursing infants from MEVACOR, women taking

Pediatric Use

Safety and effectiveness in children have not been established. Bec children are not likely to benefit from cholesterol lowering for at I decade and because experience with this drug is limited (no stud subjects below the age of 20 years.

\section{ADVERSE REACTIONS}

MEVACOR* (Lovastatin, MSD) is generally well tolerated; ad reactions usually have been mild and transient. Less than $1 \%$ of pat were discontinued from controlled clinical studies due to adverse ex ences attributable to MEVACOR. About $2 \%$ of patients were discont from all studies (controlled and uncontrolled) due to adverse experie tinued due to increases in serum transaminases.

Clinical Adverse Experiences

Adverse experiences reported in patients treated with MEVACC Antrolled clinical studies are shown in the table below:

$\begin{array}{llll}\text { MEVACOR } & \text { Placebo } & \text { Cholestyramine } & \text { Pro } \\ (\mathrm{N}=613) & (\mathrm{N}=82) & (\mathrm{N}=88) & (\mathrm{N}\end{array}$

\begin{tabular}{|c|c|c|c|}
\hline \multirow{2}{*}{\multicolumn{4}{|c|}{ Gastrointestinal }} \\
\hline & & & \\
\hline Diarthea & 5.5 & $\overline{4.9}$ & 8.0 \\
\hline Dyspepsia & 3.9 & & 13.6 \\
\hline Flatus & 6.4 & 2.4 & 21.6 \\
\hline Abdominal pain/cramps & 5. & 2.4 & 5.7 \\
\hline Heartburn & 1.6 & & \\
\hline $\begin{array}{c}\text { Nausea } \\
\text { Musculoskeletal }\end{array}$ & & 3.7 & \\
\hline Muscle cramps & 1.1 & - & 1.1 \\
\hline Myalgia & 2.4 & 1.2 & 1.1 \\
\hline $\begin{array}{l}\text { Nervous System/Psychiatric } \\
\text { Dizziness }\end{array}$ & & & \\
\hline $\begin{array}{l}\text { Hiziniess } \\
\text { Headache }\end{array}$ & $\begin{array}{l}2.0 \\
9.3\end{array}$ & 4.9 & $\overline{4.5}$ \\
\hline Rash/pruritus & 5.2 & - & 4.5 \\
\hline $\begin{array}{l}\text { Ppecial Senses } \\
\text { Blurred vision }\end{array}$ & 1.5 & - & \\
\hline Dysgeusia & 0.8 & - & $i$ \\
\hline
\end{tabular}

\section{Laboratory Tests}

Marked persistent
(see WARNINGS)

About $11 \%$ of patients had elevations of creatine phosphokase evels of at least twice the normal value on one or more occasion corresponding values for the control agents were cholestyramine. 9 probucol, $2 \%$. This was attributable to the noncardiac fraction of arge increases in CPK have rarely been reported (see WARN

In controlled clinical studies in which lovastatin was adminis concomitantly with cholestyramine, no adverse reactions peculiar concomitant treatment were observed. The adverse reactions estyramine limited to those reported previously with lovastatin or tantly with lovastatin during controlled clinical studies. In uncon clinical studies, most of the patients who have developed myopathy receiving concomitant therapy with immunosuppressive drugs, Uncontrolled Clinical Studies

The adverse experiences observed in uncontrolled studies were s were observed at a higher incidence than in the controlled studies WARNINGS, Liver Dysfunction). Myopathy (myalgia with marke elevations) was reported in approximately $0.5 \%$ of patients (see W Causal Relationship Unclear

Nervous System: A single case of peripheral neuropathy has reported: the relationship to lovastatin is uncertain. Visual evoke sponse, nerve conduction measurements, and electromyography in
30 patients showed no evidence of neurotoxic effects of lovastatin Special Senses: 01431 patients examined with slit lamp at baselin during therapy with lovastatin, 34 had opacities reported at the examination (5 to 15 months affer starting lovastatin) that were not at baseline. On the other hand, in 45 patients, opacities observe baseline were not noted at the final exar

\section{OVERDOSAGE}

The oral $\mathrm{LD}_{50}$ of MEVACOR in mice is $20 \mathrm{~g} / \mathrm{kg}$

Five healthy human volunteers have received up to $200 \mathrm{mg}$ of lovas as a single dose without clinically significant adverse experiences. A cases of accidental overdosage have been reported: no patients had
specific symptoms, and all patients recovered without sequelae. maximum dose taken was 52 20-mg tablets (1.04 g)

Until further experience is obtained, no specific treatment of dosage with MEVACOR can be recommended.

The dialyzability of lovastatin and its metabolites in man is not kno present.

\section{DOSAGE AND ADMINISTRATION}

The patient should be placed on a standard cholesterol-lowerin before receiving MEVACOR and should continue on this diet d
treatment with MEVACOR. MEVACOR should be given with meals. The recommended starting dose is $20 \mathrm{mg}$ once a day given with
evening meal. The recommended dosing range is 20 to $80 \mathrm{mg} / \mathrm{d}$ single or divided doses; the maximum recommended dose is $80 \mathrm{mg}$ Adjustments of dosage should be made at intervals of 4 weeks or $m$ Doses should be individualized according to the patient's response
Tables I to IV under CUINICAL PHARMACOL OGY, Clinical Studies for esponse results)

$300 \mathrm{mg} / \mathrm{dL}$ [ $7.8 \mathrm{mmol} / \mathrm{L}]$ on diet). MEVACOR may be initiated $\mathrm{mg} / \mathrm{daj}$

In patients taking immunosuppressive drugs concomitantly with statin (see WARNINGS,

Cholesterol levels should be monitored periodically and consider should be given to reducing the dosage of MEVACOR if cholesterol Ie fall below the targeted range.

\section{HOW SUPPLIED}

Tablets MEVACOR $20 \mathrm{mg}$ are light blue, octagonal tablets, coded use bottles of 60 and in unit-dose packages of 100 .

For more detailed information, consult your MSD Representative or 